\title{
Cerebral Revascularization Using Latissimus Dorsi Muscle Free Flap Transfer
}

\author{
-Technical Note-
}

\author{
Nobutaka YOSHIOKA and Shinsuke TOMINAGA
}

\begin{abstract}
Department of Plastic and Reconstructive Surgery, Osaka City University Medical School, Osaka; *Department of Neurosurgery, Tominaga Neurosurgical Hospital, Osaka
\end{abstract}

\begin{abstract}
A method for cerebral revascularization using latissimus dorsi muscle free flap transfer is described. Latissimus dorsi muscle is harvested with thoracodorsal vessels after completion of craniotomy and exposure of superficial temporal vessels. The size of muscle belly which is harvested is dependent on the area where the revascularization is necessary. Microvascular anastomoses of superficial temporal vessels and thoracodorsal vessels are performed in end-to-end fashion. A 31-year-old male patient with moyamoya syndrome was treated by this method. Postoperative angiography demonstrated successful revascularization in the affected hemisphere, with improved perfusion reserve capacity shown by cerebral blood flow study. This type of encephalo-myo-synangiosis (with free muscle flap) is a possible method when vascular reconstruction of an extensive ischemic area which involves anterior and posterior cerebral artery territories is needed in a patient with moyamoya disease or other similar ischemic conditions.
\end{abstract}

Key words: latissimus dorsi muscle flap, cerebral revascularization, encephalo-myo-synangiosis, moyamoya syndrome, surgical treatment, free flap

\section{Introduction}

The most widely accepted treatment for moyamoya disease seems to be surgical reconstruction to increase collateral circulation and decrease hemodynamic stress in the basal moyamoya vessels. ${ }^{4-6,9,10,13)}$ Surgical treatment involves direct and indirect revascularization. Omental transplantation is effective, especially for ischemic symptoms in the territories of the anterior cerebral artery (ACA) and posterior cerebral artery. ${ }^{2,3,7,8)}$ Recently, Touho et al. ${ }^{12)}$ reported the effectiveness of gracilis muscle transplantation for the childhood moyamoya disease. We describe a modified encephalo-myo-synangiosis (EMS) using latissimus dorsi muscle free flap in a patient with moyamoya syndrome.

\section{Clinical Course and Operative Technique}

A 31-year-old male developed acute left hemimotor

Received May 16, 1996; Accepted November 20, 1996 weakness on May 23,1994. This symptom gradually improved and almost disappeared 7 days after onset. Computed tomography (CT) demonstrated a watershed infarction measuring $2 \times 1 \mathrm{~cm}$ in the right frontal lobe. Cerebral angiography demonstrated occlusion of the proximal segment of the right middle cerebral artery (MCA) and compensating moyamoya vessels. Narrowing of the left MCA and ACA was also demonstrated but this was not significant (Fig. 1). The cerebral blood flow (CBF) was qualitatively evaluated using intravenous injection of $\mathrm{N}$ isopropyl-p-(I-123) iodoamphetamine $\left({ }^{123} \mathrm{I}-\mathrm{IMP}\right) \mathrm{com}$ bined with single photon emission computed tomography (SPECT). Vasoreactivity was also measured 15 minutes after intravenous injection of 15 $\mathrm{mg} / \mathrm{kg}$ acetazolamide. ${ }^{123}$ I-IMP SPECT revealed reduced perfusion reserve capacity in the right frontal, temporal, and parietal lobes (Fig. 2).

On June 23, 1994 latissimus dorsi muscle free flap transfer to the right cerebral surface was performed to revascularize the area in which the perfusion reserve capacity was impaired. The patient was placed in the right lateral decubitus position with 


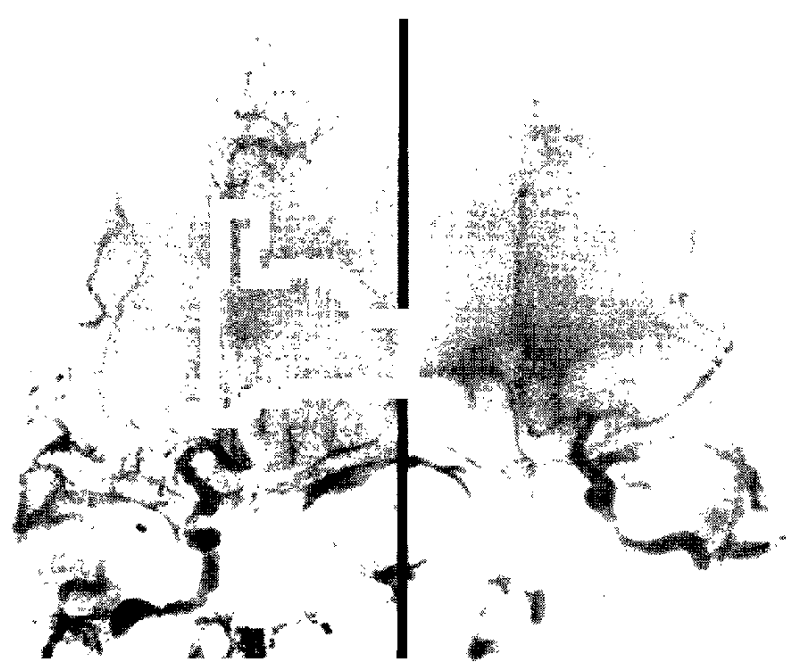

Fig. 1 Preoperative right (left) and left (right) common carotid angiograms, showing the right middle cerebral artery was occluded, moyamoya vessels, and mild stenosis of the left anterior and middle cerebral arteries.

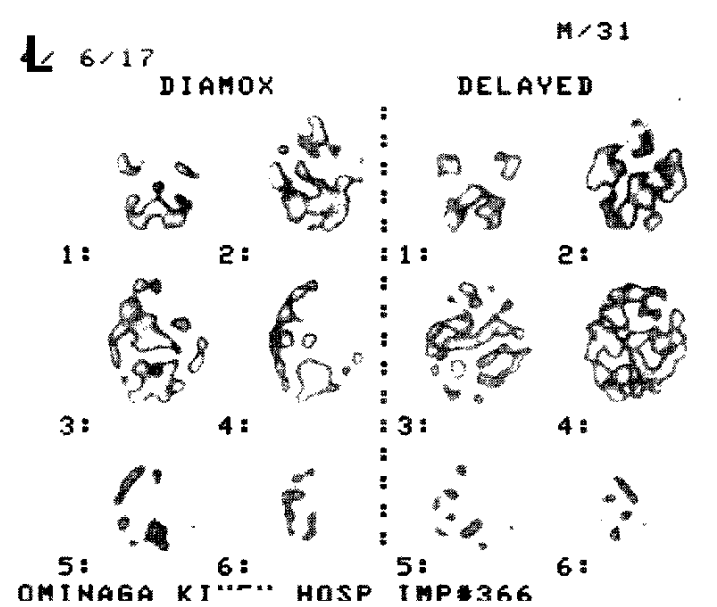

228 $87 \mathrm{~nm}$

295

$18 c$

159

$\mathbf{R}$

136

113

96

Fig. 2 N-isopropyl-p-(I-123) iodoamphetamine single photon emission computed tomography scans after acetazolamide administration revealing reduced perfusion reserve capacity of the right cerebral hemisphere.

three point pin fixation. Initially the superficial temporal artery (STA) and vein (STV) were dissected and prepared for microvascular anastomosis in the preauricular region. Then an $\mathrm{H}$-shaped skin incision was made on the head. Craniotomy was performed and the dura opened. The arachnoid membrane was cut at several sulci. Simultaneously, the latissimus dorsi muscle was elevated. The size of muscle belly which was harvested was matched to the area where


Fig. 3 Schema of Iatissimus dorsi muscle elevation showing sacrifice of the thoracodorsal nerve (upper), and intraoperative view after microvascular anastomosis (lower) showing the monitoring segment (arrowhead) and the thoracodorsal vein (arrow). Both the skin and temporal muscle are cut and separated on the vascular pedicle to prevent disturbance of the vessels.

the revascularization is necessary. After preparation of the recipient site, the latissimus dorsi muscle was harvested free with thoracodorsal vessels. The donor site was closed primarily with a suction drain. The latissimus dorsi muscle was trimmed, spread over the brain, and attached to the dura with mattress sutures. The surface attached to the brain was the non bleeding surface and contained nutrient vessels with a thin fatty layer. The STA and STV were end-to-end anastomosed to the thoracodorsal artery and vein using 10-0 nylon sutures. Approximately 10 to 12 stitches were necessary in each vessel. Ischemic time was about 2 hours. The thickness of the latissimus dorsi muscle is sometimes bulky in adult male patients. Therefore, the muscle belly was 


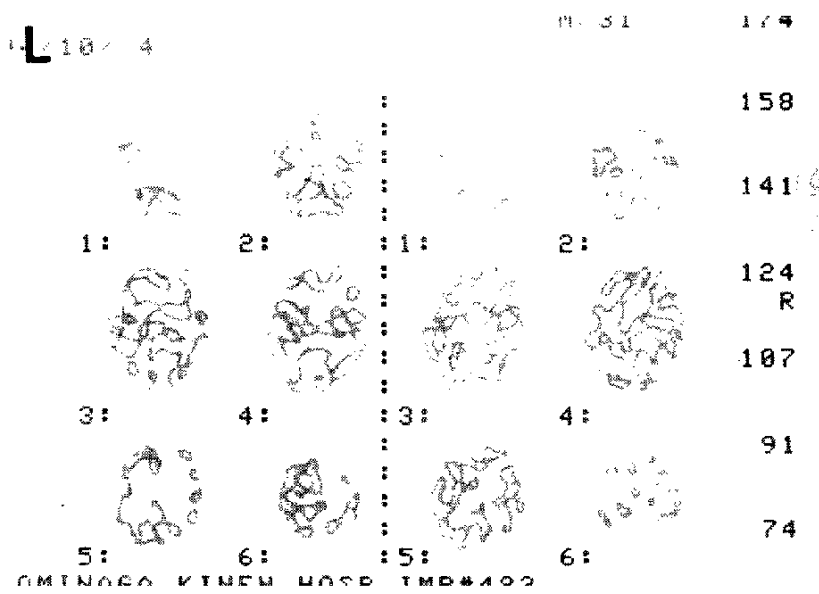

Fig. 4 Postoperative N-isopropyl-p-(I-123) iodoamphetamine single photon emission computed tomography scans after acetazolamide administration revealing improved perfusion reserve capacity of the right cerebral hemisphere.

shaved on the superficial side in this case using a electrocoagulator. Then titanium cranioplasty was applied to prevent the mass effect due to muscle swelling. The distal parts of the latissimus dorsi muscle were exteriorized to monitor the muscle circulation. Total operation time was 7 hours. For early detection of occlusion of the anastomotic site, the segment was monitored daily by pin-prick test. Approximately 7 days after surgery, the monitoring segment was cut and coagulated (Fig. 3).

The postoperative course was uneventful. CBF study 3 months after surgery revealed improvement of the perfusion reserve capacity in the right cerebral hemisphere (Fig. 4). Selective external carotid angiography 7 months after surgery disclosed moderately collateral circulation from the latissimus dorsi muscle to the brain (Fig. 5). CT did not demonstrate any mass effect from the transferred muscle flap (Fig. 6). The patient did not experience any further symptoms after surgery.

\section{Discussion}

Both the omentum and muscle are considered richly vascular tissues, and many applications have been reported in plastic and reconstructive surgery. ${ }^{1,11)}$ We had already performed EMS with serratus anterior muscle free flap (EMS with free muscle flap) in a previous patient, which proved to be as effective as an omental flap. EMS with free muscle flap does not require laparotomy and has several advantages over EMS with temporal muscle. ${ }^{14)}$ Latissimus dorsi muscle is larger than serratus anterior muscle and both muscles can be harvested easily. Therefore, we used EMS with latissimus dorsi muscle free flap in this patient. The deep surface of this muscle is covered with a thin fatty layer containing the nutrient vessels, which separates the serratus anterior muscle and latissimus dorsi muscle. This deep surface was attached to the brain surface in our case. The thickness of the latissimus dorsi muscle is sometimes bulky in adult male patients. Therefore, the muscle belly was shaved using a electrocoagulator. Although we applied artificial cranioplasty in this patient, we are currently thinning the calvarial bone flap in other patients, if needed.

CBF study and angiography revealed improved collateral circulation after the surgery. CT revealed no mass effect of the latissimus dorsi muscle flap and
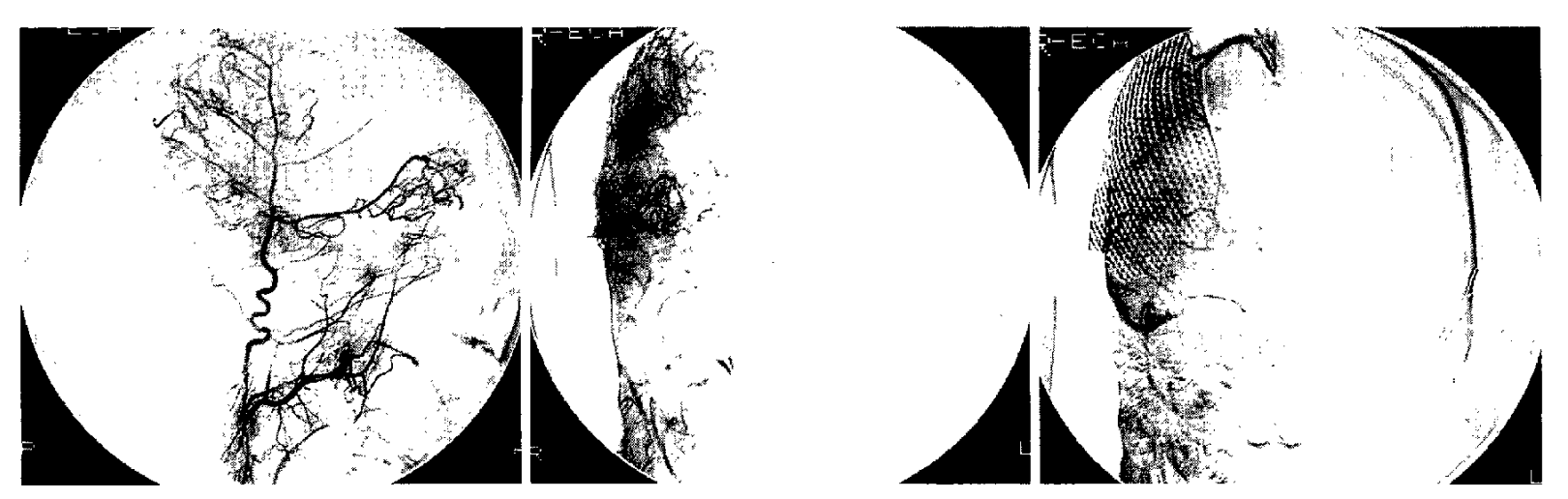

Fig. 5 Postoperative right selective external carotid angiograms, lateral (left) and anteroposterior views (center) during the arterial phase, and the anteroposterior view during the capillary to venous phase (right), showing the right cortical arteries through the transferred latissimus dorsi muscle, and the ascending vein. 

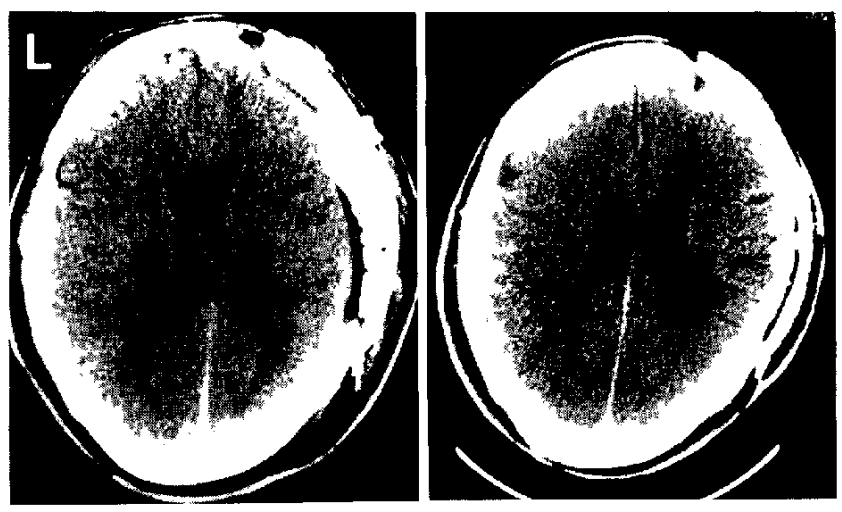

Fig. 6 Computed tomography scans 1 day (left) and 8 months after the operation (right) revealing no mass effect due to the latissimus dorsi muscle flap in the right cerebral hemisphere.

no symptoms related to increased intracranial pressure developed. The latissimus dorsi muscle was as useful as the serratus anterior muscle, and harvesting of this muscle was associated with minimal complications.

We found that EMS with latissimus dorsi muscle free flap is effective for the treatment of moyamoya syndrome. This EMS method is a possible method for the vascular reconstruction of an extensive ischemic area which involves the ACA and posterior cerebral artery territories in a patient with moyamoya disease or other similar ischemic conditions.

\section{References}

1) Hammond DC, Fisher J, Meland B: Intrathoracic free flaps. Plast Reconstr Surg 91: 1259-1264, 1993

2) Ichikawa $A$, Tanaka $R$, Takeuchi $S$, Koike $T$, Ishii R: [Reconstructive vascular surgery in the territory of the anterior cerebral artery in children with moyamoya disease]. Neurol Med Chir (Tokyo) 29: 106-112, 1989 (Jpn)

3) Karasawa J, Touho H, Ohnishi H, Miyamoto S, Kikuchi H: Cerebral revascularization using omental transplantation for childhood moyamoya disease. J Neurosurg 79: 192-196, 1993
4) Matsushima T, Inoue T, Suzuki SO, Fujii K, Fukui M, Hasuo K: Surgical treatment of moyamoya disease in pediatric patients: Comparison between the results of indirect and direct revascularization procedures. Neurosurgery 31: 401-405, 1992

5) Matsushima Y: [Facts and myths in indirect extracranial to intracranial cerebrovascular bypass operation as a treatment of moyamoya disease]. Shoni No Noshinkei 17: 219-229, 1992 (Jpn)

6) Matsushima Y, Suzuki R, Yamaguchi T, Tabata H, Inaba Y: [Effects of indirect EC/IC bypass operations on adult moyamoya patients]. No Shinkei Geka 14: 1559-1566, 1986 (Jpn)

7) Miyamoto S, Kikuchi H, Karasawa J, Nagata I, Ihara I, Yamagata S: Study of the posterior circulation in moyamoya disease. Part 2: Visual disturbances and surgical treatment. J Neurosurg 65: 454-460, 1986

8) Miyamoto S, Kukuchi H, Karasawa J, Nagata I, Yamazoe N, Akiyama Y: Pitfalls in the surgical treatment of moyamoya disease. J Neurosurg 68: 537-543, 1988

9) Nakagawa $Y$, Abe H, Sawamura $Y$, Kamiyama H, Gotoh S, Kashiwaba T: Revascularization surgery for moyamoya disease. Neurol Res 10: 32-39, 1988

10) Olds MV, Griebel RW, Hoffman HJ, Craven M, Chuang S, Schutz H: The surgical treatment of childhood moyamoya disease. J Neurosurg 66: 675-680, 1987

11) Ringelman PR, Vander Kolk CA, Cameron D, Baumgartner WA, Manson PN: Long-term results of flap reconstruction in median sternotomy wound infections. Plast Reconstr Surg 93: 1208-1216, 1994

12) Touho H, Karasawa J, Ohnishi H: Cerebral revascularization using gracilis muscle transplantation for childhood moyamoya disease. Surg Neurol 43: 191198, 1995

13) Wanifuchi $H$, Takeshita $M$, Izawa $M$, Aoki $N$, Kagawa M: Management of adult moyamoya disease. Neurol Med Chir (Tokyo) 33: 300-305, 1993

14) Yoshioka N, Tominaga S, Inui T: Cerebral revascularization using omentum and serratus anterior muscle free flap transfer for adult moyamoya disease: Case report. Surg Neurol 46: 430-436, 1996

Address reprint requests to: N. Yoshioka, M.D., Department of Plastic and Reconstructive Surgery, Osaka City University Medical School, 1-5-7 Asahi-machi, Abeno-ku, Osaka 545, Japan. 